\title{
Response to the letter to the Editor: FKBP5 polymorphism rs1360780 and weight loss after bariatric surgery
}

We have read with attention the letter of Yasri and Wiwanitkit. According to our recent paper [1], as stated by the authors, our results suggest how age, sex and FKBP5 genotype have a different impact on weight loss depending on the surgical technique used, sex and age. These factors could contribute to understand the interindividual variation in surgery outcomes, especially in relation to weight loss suffered by the patients in the post-operative period.

Obesity is a polygenic multifactorial trait, influenced by multiple genetic variants of minor effect and environmental factors. Recent Genome-Wide Association Studies, have identified a number of common loci associated with obesity-related phenotypes (e.g: BMI or waist circumference), although most of the genetic variability for BMI remains unexplained [2]. However, the association of these variants with weight loss after bariatric surgery has been less examined. In this regard, Hartman's work and our paper, published in this journal, analysed a single functional polymorphism (rs1360780) and reported the possible contribution of the protein encoded by FKBP5 gene in metabolic regulation.

Kops and colleagues [3], cited by Yasri and Wiwanitkit, also analysed the variability in four obesity-related genes (LEP223, LEP656, FTO and FABP2) in relation to their effects on weight loss after bariatric surgery. They found a different change on weight loss in those patients with AA genotype in the LEP223 gene (rs1137101). These studies are contributing to understand how the biological background, among other factors, impact in the outcomes after bariatric surgery.

As commented in our paper, we only studied a candidate gene and we cannot discard the effect of other genetic markers via complex interactions with environmental factors. Future studies should consider polygenic scores taking into account the variation and risk conferred by multiple genetic variants. These scores could have a better predictive power in relation to the evolution of the obese patients after surgery.

\section{References:}


[1] Pena E, Caixas A, Arenas C, Rigla M, Crivilles S, Cardoner N, et al. Role of the FKBP5 polymorphism rs1360780, age, sex, and type of surgery in weight loss after bariatric surgery: a follow-up study. Obes Relat Dis 2020;16(4):581-9.

[2] Thorleifsson G, Walters GB, Gudbjartsson DF, et al. Genome-wide association yields new sequence variants at seven loci that associate with measures of obesity. Nat Genet 2009;41(1):18-24.

[3] Hartmann IB, Fries GR, B€ucker J, Scotton E, von Diemen L, KauerSant'Anna M. The FKBP5 polymorphism rs 1360780 is associated with lower weight loss after bariatric surgery: 26 months of followup.Surg Obes Relat Dis 2016;12(8):1554-60.

[4] Kops NL, Vivan MA, Horvath JDC, de Castro MLD, Friedman R.FABP2, LEPR223, LEP656, and FTO polymorphisms: effect on weight loss 2 years after bariatric surgery. Obes Surg 2018;28(9):2705-11.Surg Obes Relat Dis 2019. https://doi.org/10.1016/j.soard.2019.12.002. 